[11] R. Sedgewick, Data movement in odd-even merging, SIAM J. Comput. 7 (1978), 239-272.

2] K. B. Stolarsky, Power and exponential sums related to binomial digit parity, SIAM J. Appl. Math. 32 (1977), 717-730. [13] R. F. Tichy and G. Turnwald, On the discrepancy of some special sequences, J. Number
Theory 26 (1987), 68-78.

[14] -, - Gleichmässige Diskrepanzabschätzung fir Ziffernsummen, Anz. Osterr. Akad. Wiss. (1986), 17-21.

MATHEMATISCHES INSTITUT

DER UNIVERSITÄT SALZBURG

Hellbrunner Strasse 34

ABTEILUNG FOR TECHNISCHE MATHEMATIK

TU WIEN

Wiedner Hauptstrasse 8-10

A-1040 Wien, Austria

\section{On the number of values taken by a polynomial over a finite field}

by

J. F. Voloch (Rio de Janeiro)

Let $\boldsymbol{F}_{q}$ be the finite field with $q$ elements and $f(x) \in \boldsymbol{F}_{q}[x]$ a polynomial of degree $n$. Let $r(f)=\# f\left(\boldsymbol{F}_{q}\right)$, considering $f$ as a function $f: \boldsymbol{F}_{q} \rightarrow \boldsymbol{F}_{q}$. A classical problem, raised by Chowla [3] (see [4] for other references), is to estimate $r(f)$ an terms of $n$ and $q$. One has the trivial bounds $q / n \leqslant r(f) \leqslant q$. The lower bound is essentially best possible and a characterization of the cases with equality when $q$ is prime was obtained in [2].

On the other hand, if $f$ is a "general" polynomial (in a sense that can be made precise, see below) Uchiyama [6] proved that $r(f) \geqslant q / 2+O\left(q^{1 / 2}\right)$ and Birch and Swinnerton-Dyer [1] found the precise result

$$
r(f)=q\left(\sum_{i=1}^{n} \frac{(-1)^{i-1}}{i !}\right)+O\left(q^{1 / 2}\right) .
$$

They proved this when the Galois group of $f(x)=y$ over $\overline{\boldsymbol{F}}_{q}(y)$ is the full symmetric group. Of course these results are interesting only when $q$ is large compared to $n$. The purpose of this paper is to give lower bounds for $r(f)$. valid for $f$ "general", which improves on the above bounds in several cases.

Uchiyama's condition is that the polynomial

$$
f^{*}(u, v)=(f(u)-f(v)) /(u-v)
$$

is absolutely irreducible. When this is the case he could apply Weil's estimate ([7]) on the number of points of $f^{*}(u, v)=0$ over $\boldsymbol{F}_{q}$ to get his result.

To relate the number of solutions of $f^{*}(u, v)=0$ in $\boldsymbol{F}_{q}^{2}$ with $r(f)$, Uchiyama [6] proved the following:

Lemma 1. Let $N$ be the number of solutions of $f^{*}(u, v)=0$ in $F_{q}^{2}$ and $n_{0}$ the number of solutions of $f^{\prime}(x)=0$ in $\boldsymbol{F}_{q}$. Then

$$
r(f) \geqslant q^{2} /\left(N+q-n_{0}\right) \text {. }
$$

Proof. First notice that $f^{*}(u, v)=0$ and $u \neq v$ if and only if $f(u)=f(v)$ and that $f^{*}(u, u)=f^{\prime}(u)$. Let $\left\{a_{1}, \ldots, a_{r}\right\}=f\left(F_{q}\right), \quad r=r(f)$ and $n_{i}$ 
$=\# f^{-1}\left(a_{i}\right), i=1, \ldots, r$. Then $\sum_{i=1}^{r} n_{i}=q$ and

$$
N=\sum_{i=1}^{r} n_{i}\left(n_{i}-1\right)+n_{0} .
$$

Hence, $\sum_{i=1}^{r} n_{i}^{2}=N+q-n_{0}$. By the Cauchy-Schwarz inequality

$$
\sum_{i=1}^{r} n_{i}^{2} \geqslant \frac{1}{r}\left(\sum n_{i}\right)^{2}=\frac{q^{2}}{r}
$$

and the result follows.

Using the trivial bound $N \leqslant(n-1) q$ (since $f^{*}$ has degree $n-1$ ) one gets $r(f) \geqslant q / n$. If $f^{*}$ is absolutely irreducible (i.e. irreducible over $\overline{\boldsymbol{F}}_{q}$ ), Weil's estimate $N \leqslant q+(n-3)(n-2)\left(q^{1 / 2}+1\right)$ gives

$$
r(f) \geqslant \frac{q}{2}-\frac{(n-3)(n-2)\left(q^{1 / 2}+1\right)}{4} .
$$

We shall now give upper bounds for $N$ which follow from the results of [5] and improve on the above bounds on several instances.

THEOREM. Let $X$ be an absolutely irreducible plane curve of degree $d$ defined over $\boldsymbol{F}_{q}$ with $N$ rational points, then

(i) If $q$ is prime and $q^{1 / 4}<d<q$ then $N \leqslant 4 d^{4 / 3} q^{2 / 3}$.

(ii) If $h(x, y)=0$ is an affine equation for $X$ and $d^{2} y / d x^{2} \neq 0$, then $N \leqslant \frac{1}{2} d(d+q-1)$.

Proof. (i) Let $X$ be an absolutely irreducible curve of degree $D$ contained in $\boldsymbol{P}^{n}$, not contained in a hyperplane. If $p$ is the characteristic of $\boldsymbol{F}_{q}$ and $D \leqslant p$, it follows from [5], Theorem 2.13 and Corollary 2.7, that the number of rational points, $M$ say, of a non-singular model of $X$ satisfies

$$
M \leqslant(n-1)(g-1)+D(q+n) / n
$$

where $g$ is the genus of $X$.

Returning to the situation of the theorem, let $x, y$ be affine coordinates in the plane. If $m<d$, we can embed $X$ in $\boldsymbol{P}^{n}, n=\left(\begin{array}{c}m+2 \\ 2\end{array}\right)-1$ by $(x, y) \mapsto(x, y$, $\left.x^{2}, x y, y^{2}, \ldots, x^{m}, \ldots, y^{m}\right)$ in affine coordinates. In this case $D=m d$, and this embedding is not contained in a hyperplane, so we can apply the above bound if $D \leqslant p$. Now the number of singular points of $X$ is bounded by $(d-1)(d-2) / 2-g$, hence for $m<d$ and $D \leqslant p$ we get

$$
N \leqslant(n-1) \frac{d(d-3)}{2}+\frac{D(q+n)}{n}
$$

with $n=\left(\begin{array}{c}m+2 \\ 2\end{array}\right)-1, D=m d$.

If we take now $m=\left[(q / d)^{1 / 3}\right]$, the conditions $m<d$ and $D \leqslant p$ follow from the hypotheses $q^{1 / 4}<d<q$ and $q=p$, and the result stated follows immediately.

(ii) is just Theorem 0.1 of [5].

Applying item (i) of the theorem to $f^{*}(u, v)=0$ when it is absolutely irreducible, it follows that $r(f) \geqslant \frac{1}{4}\left(\frac{q}{n-1}\right)^{4 / 3}$, if $q$ is prime and $q^{1 / 4}<n-1$ $<q$. In this range this bound is better than those mentioned above.

Whenever (ii) applies, it gives

$$
r(f) \geqslant \frac{2 q^{2}}{(n+1) q+(n-1)(n-2)}
$$

which improves on Uchiyama's bound for $n>q^{1 / 2} / 2$.

We shall now study when the conditions $f^{*}(u, v)$ absolutely irreducible and $d^{2} v / d u^{2} \neq 0$ on $f^{*}(u, v)=0$, hold. Consider the following condition on $f$ :

(*) $f^{\prime}$ has $n-1$ distinct roots and $f$ is injective on the roots of $f^{\prime}$.

This condition already appears in [1]. There they prove that $(*)$ is sufficient for the Galois group of $f(x)=y$ over $\overline{\boldsymbol{F}}_{q}(y)$ to be the full symmetric group ([1], Lemma 3). They also remark that $(*)$ is equivalent to the nonvanishing of the discriminant in $y$ of the discriminant in $x$ of $f(x)-y$. The aforementioned discriminant is a function on the coefficients of $f$, which does not vanish identically if $p \neq 2$ and $p \nmid n$, where $p$ is the characteristic of $\boldsymbol{F}_{q}$. Hence $(*)$ is a generic condition.

Concerning condition (*) we shall prove

Proposition. Suppose that the characteristic of $F_{q}$ is not 2 and let $f(x) \in F_{q}[x]$ be of degree $n \geqslant 2$.

(i) $f^{*}(u, v)=0$ is non-singular if and only if $f$ satisties $(*)$.

(ii) If $f$ satisfies (*) then, on $f^{*}(u, v)=0, d^{2} v / d u^{2} \neq 0$.

Proof. (i) Let $p$ be the characteristic of $\boldsymbol{F}_{q}$. If $p \nmid n$ it is easy to see that $f^{*}(u, v)=0$ has $n-1$ points at infinity, hence they are all non-singular points. If $p \mid n$ it is also easy to see that the point at infinity on the line $u=v$ is a singular point of $f^{*}(u, v)=0$. Also condition $(*)$ implies that $p \nmid n$, for otherwise $f^{\prime}$ would have degree at most $n-2$. This takes care of the points at infinity. 
For the affine points, we have:

$$
\begin{aligned}
& \frac{\partial f^{*}}{\partial u}=\frac{(u-v) f^{\prime}(u)-(f(u)-f(v))}{(u-v)^{2}}, \\
& \frac{\partial f^{*}}{\partial v}=\frac{-(u-v) f^{\prime}(v)+f(u)-f(v)}{(u-v)^{2}} .
\end{aligned}
$$

A point $\left(u_{0}, v_{0}\right)$ with $u_{0} \neq v_{0}$ is in $f^{*}(u, v)=0$ if and only if $f\left(u_{0}\right)=f\left(v_{0}\right)$ and is a singular point if and only if $f^{\prime}\left(u_{0}\right)=f^{\prime}\left(v_{0}\right)=0$, in which case $f$ is not injective on the set of zeros of $f^{\prime}(x)=0$

Let now $\left(u_{0}, u_{0}\right)$ be a point of $f^{*}(u, v)=0$. Changing variables, $x$ to $x+u_{0}, u$ to $u+u_{0}, v$ to $v+u_{0}$, we may assume that $u_{0}=0$ and $f^{\prime}(0)=0$. If $f(x)=\sum_{i=0}^{n} \alpha_{i} x^{i}$, then $\alpha_{1}=0$ and

$$
f^{*}(u, v)=\alpha_{2}(u+v)+\alpha_{3}\left(u^{2}+u v+v^{2}\right)+\ldots
$$

Hence $(0,0)$ is a singular point of $f^{*}=0$ if and only if $\alpha_{2}=0$, which is equivalent to $x=0$ be a double root of $f^{\prime}(x)=0$. This proves part (i) of the proposition.

(ii) On $f^{*}(u, v)=0$ we have $f(u)=f(v)$, hence $f^{\prime}(u)=f^{\prime}(v) d v / d u$ and

$$
f^{\prime \prime}(u)=f^{\prime \prime}(v)(d v / d u)^{2}+f^{\prime}(v) d^{2} v / d u^{2} .
$$

If $d^{2} v / d u^{2}=0$ we conclude that $f^{\prime \prime}(u) \cdot f^{\prime}(v)^{2}=f^{\prime \prime}(v) f^{\prime}(u)^{2}$, whenever $f(u)=f(v)$. Suppose $f$ satisfies $(*)$. Let $\alpha$ be a root of $f^{\prime}(x)=0$. Since $(*)$ holds there exists $\beta \neq \alpha$ with $f(\beta)=f(\alpha)$. Then

$$
f^{\prime \prime}(\alpha) f^{\prime}(\beta)^{2}=f^{\prime \prime}(\beta) f^{\prime}(\alpha)^{2}=0 .
$$

If $f^{\prime \prime}(\alpha)=0, \alpha$ is a double root of $f^{\prime}(x)=0$, contradicting $(*)$. If $f^{\prime}(\beta)=0$ then $f$ is not injective on the roots of $f^{\prime}(x)=0$, again contradicting (*). This completes the proof of the proposition.

Remarks. 1. A non-singular plane curve is necessarily absolutely irreducible, since two irreducible components would necessarily meet at a singular point. Hence $f^{*}=0$ is absolutely irreducible when $(*)$ holds.

2. It follows from item (ii) of the proposition that item (ii) of the theorem holds for $f^{*}$ whenever $(*)$ holds for $f$ and, in this case, we have the corresponding bound on $r(f)$.

\section{References}

[1] B. J. Birch and H. P. F. Swinnerton-Dyer, Note on a problem of Chowla, Acta Arith. 5 (1959), 417-423.

[2] L. Carlitz, D. J. Lewis, W. H. Mills and E. G. Straus, Polynomials over finite field with minimum ralue sets, Mathematika 8 (1961), 121-130.
[3] S. Chowla, The Riemann zeta and allied functions, Bull. Amer. Math. Soc. 58 (1952), 287305.

[4] R. Lidl and H. Niederreiter, Finite Fields, Addison-Wesley, Reading, Mass., 1983.

[5] K. O. St öhr and J. F. Voloch, Weierstrass points and curves over finite fields, Proc. London Math. Soc. (3) 52 (1986), 1-19.

[6] S. Uchiyama, Sur le nombre des valeurs distinctes d'un polynome à coefficients dans un corps fini, Proc. Japan Acad. 30 (1955), 930-933.

[7] A. Weil, Sur les courbes algébriques et variétés qui s'en déduisent, Hermann, Paris 1948.

IMPA

Estrada Dona Castorina 110

22460 - Rio de Janeiro. $R$

Brazil 